\title{
A Method to Extend the Spectral Iteration Technique
}

\author{
S. A. BOKHARI AND N. BALAKRISHNAN
}

\begin{abstract}
A method has been developed to extend the spectral iteration technique to encompass a more general class of subdomain type basis functions. While retaining the advantages of the spectral iteration technique, this method enables the usage of basis functions other than piecewise constant basis functions in expressing the unknown current distribution. Advantages of this technique with the choice of piecewise sinusoidal basis functions as compared to piecewise constant basis functions have been demonstrated for both antenna and scattering problems. This choice of basis functions is found to result in a further reduction in the number of unknowns required to represent the current distribution. Numerical results of the input impedance for a cylindrical antenna and the scattering cross section of a thin flat plate are presented and discussed.
\end{abstract}

\section{INTRODUCTION}

$\mathrm{T}$ HE LIMITATIONS of conventional numerical methods in handling electromagnetic radiation and scattering problems in the intermediate or resonance frequency range have long been recognized. An excellent review of techniques applicable to problems in this range is given by Thiele [1]. Among the iterative methods proposed for solving problems in this intermediate range the spectral iteration technique (SIT) [2], the conjugate gradient method [3], and a modified steepest descent technique in the Fourier transform domain [4] have been found to hold considerable promise. These techniques are not merely iterative methods of solving the matrix equation but involve a unique kind of processing which does away with the need to store the generalized impedance matrix arising in moment method solutions. The SIT, like other iterative methods, has a provision to check for the satisfaction of the boundary conditions. This technique has been successfully applied to a variety of scattering problems [5], [6]. However, in order to assess the accuracy of this technique, it is important to consider the basis functions that are used to represent the current distribution as well as the testing procedure employed in the solution. The SIT for conducting scatterers begins by taking the Fourier transform on both sides of the electric field integral equation. This amounts to choosing cisoidal ( $\cos +j$ $\sin$ ) functions as the testing functions. Secondly, the Fourier transform of the current distribution is approximated by the discrete Fourier transform (DFT) and computed by the FFT algorithm [7]. Since the DFT is employed, assuming a piecewise constant distribution for the current is therefore inherently implied, although not explicitly stated. Hence, the

Manuscript received August 20, 1984; revised April 19, 1985.

The authors are with the Department of Aerospace Engineering, Indian Institute of Science, Bangalore 560012 , India.

IEEE Log Number 8406147.
SIT may be briefly interpreted as an efficient iterative method for the solution of the integral equation with piecewise constant basis (PWC) functions to represent the current distribution and over determined spectral matching at the boundary (see [8] for details).

In moment method solutions it is well known that both basis as well as testing functions play important roles in the accuracy and convergence of the solutions. In general, basis functions which more closely approximate the unknown current distribution in conjunction with certain testing procedures have been found to yield a relatively more rapidly covergent solution. For example, Richmond's [9] solution for thin wire antennas with piecewise sinusoidal (PWS) functions as basis functions and reaction matching has yielded results of input impedance which compare well with Harrington's [9] solution with six times as many triangular basis functions and point matching. Likewise, Newman and Pozar [10] in their analysis of monopoles on finite size ground planes using piecewise sinusoidal basis functions and reaction matching have obtained a better agreement of input impedance with experimental results as compared to that obtained using four times as many piecewise constant basis functions. Their success can be attributed partly to their choice of basis functions that more closely approximate the unknown current distribution and partly to the use of reaction matching which happens to be a physical concept. It is therefore of interest to investigate the effectiveness of other subdomain basis functions when spectral matching is used as the testing procedure.

In this paper, a method is presented to extend the spectral iteration technique to include a larger class of subdomain basis functions. Although it is indeed possible in principle to use arbitrary basis functions, the method presented in the paper achieves its purpose with a minimum of extra computations while retaining all the advantages of the SIT. Results are illustrated using piecewise sinusoidal basis functions.

\section{Formulation: One-Dimensional Current Distributions}

Consider a straight, center-fed cylindrical wire of circular cross-sectional radius $a$ and length $2 h$ symmetrically located at the origin of a rectangular coordinate system and oriented along the $z$-axis. With the assumed thin wire approximations, the Pocklington's integral equation is of the form

$$
\begin{aligned}
{\left[\frac{d^{2}}{d z^{2}}+\beta^{2}\right] \int_{-h}^{h} J\left(z^{\prime}\right) G\left(z-z^{\prime}\right) d z^{\prime} } & \\
& =-j \omega \epsilon E_{z i}(z), \quad|z| \leq h
\end{aligned}
$$


where $\beta$ and $\epsilon$ denote the propagation constant and permittivity of the medium, $E_{z i}(z)$ the tangential component of the incident electric field, $J\left(z^{\prime}\right)$ the current distribution ( $z$-directed) and $G\left(z-z^{\prime}\right)$ is the free space Green's function given by

$$
G\left(z-z^{\prime}\right)=\frac{\exp \left[-j \beta \sqrt{\left(z-z^{\prime}\right)^{2}+a^{2}}\right]}{4 \pi \sqrt{\left(z-z^{\prime}\right)^{2}+a^{2}}} .
$$

Since (1) is valid on the wire only, to enable Fourier transformation a truncation operator $\theta$ is defined as

$$
\begin{aligned}
\theta(\dot{z}) & =1, & & -h \leq z \leq h \\
& =0, & & \text { otherwise }
\end{aligned}
$$

and a complementary operator $\hat{\theta}$ is defined as

$$
\begin{aligned}
\hat{\theta}(z) & =0, & & -h \leq z \leq h \\
& =1, & & \text { otherwise. }
\end{aligned}
$$

Defining the one-dimensional Fourier transform as

$$
\tilde{H}=\tilde{H}(f)=F[H(z)]=\int_{-\infty}^{\infty} H(z) \exp (-j 2 \pi f z) d z
$$

and its inverse as

$$
H=H(z)=F^{-1}[\tilde{H}(f)]=\int_{-\infty}^{\infty} \tilde{H}(f) \exp (-j 2 \pi f z) d f
$$

an iterative expression for the current distribution is written as (see [2] and [8] for details)

$$
J^{(k+1)}=F^{-1}\left[(\tilde{D} \tilde{G})^{-1}\left\{F\left(-\theta E_{z i}\right)+F\left(\hat{\theta} F^{-1}\left(\tilde{D} \tilde{G} F\left(\theta J^{k}\right)\right)\right)\right\}\right]
$$

where

$\tilde{D}=\frac{\left[\beta^{2}-(2 \pi f)^{2}\right]}{j \omega \epsilon} ;$

$\tilde{G}=\frac{K_{0}\left[a \sqrt{(2 \pi f)^{2}-\beta^{2}}\right]}{2 \pi}, \quad k=0,1,2, \cdots$.

$K_{0}$ denotes the modified Bessel function of the second kind and zeroth order and the superscripts $k$ indicate the iteration numbers. Since the DFT is employed, the resulting current distribution obtained would be a piecewise constant approximation to the actual distribution as shown in Fig. 1, i.e., the current distribution may be written as

$$
J_{\mathrm{PWC}}=\sum_{n=-N_{p}}^{N_{p}} I_{n} P(z-n \Delta z)
$$

where

$$
\begin{aligned}
P(z) & =1, & & |z| \leq \Delta z / 2 \\
& =0, & & \text { otherwise, }
\end{aligned}
$$

the number of basis functions are $\left(2 N_{p}+1\right), \Delta z$ denotes the sampling interval or the width of the pulses, and $I_{n}$ denote the unknown coefficients that are to be determined.

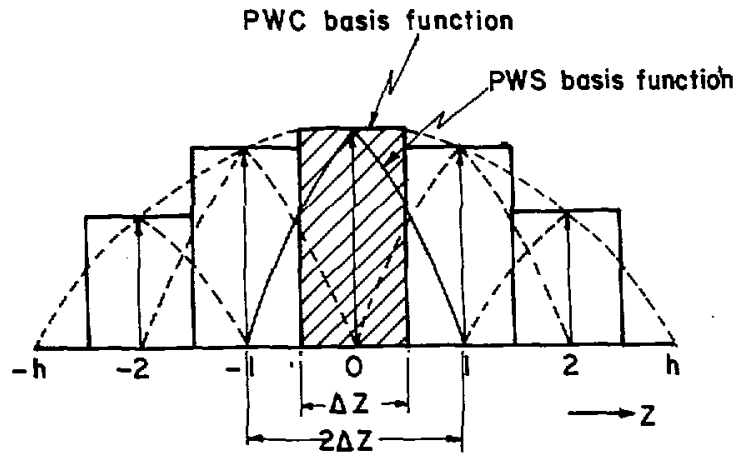

Fig. 1. Representation of the current distribtion in terms of one-dimensional piecewise constant and piecewise sinusoidal basis functions.

It can be seen from (7) that the Fourier transforms of the differential operator and the Green's function are evaluated in a closed form. The Fourier transform of the incident electric field for many wire antenna feed models as well as for plane wave scattering problems can also be computed in a closed form. Therefore, errors due to aliasing can occur only in those calculations where the FFT is employed in the computation of the Fourier transform and the inverse, namely

(a) $F\left[\theta J^{k}\right]$,

(b) $F^{-1}\left[\tilde{J}^{k+1}\right]$,

(c) $F^{-1}\left[\tilde{D} \tilde{G} F\left(\theta J^{k}\right)\right]$

(d) $F\left[\hat{\theta} F^{-1}\left\{\tilde{D} \tilde{G} F\left(\theta J^{k}\right)\right\}\right]$.

Often, one resorts to increasing the sampling rate to reduce the aliasing error, in other words increase the number of basis functions used to represent the current distribution. In the method presented in this paper we reduce the errors in cases (a) and (b) above by using a more appropriate basis function to represent the current distribution rather than by increasing the number of basis functions.

Let the current distribution be represented in terms of an overlapping set of piecewise sinusoidal basis functions as shown in Fig. 1, i.e.,

$$
J_{\mathrm{PWS}}=\sum_{n=-N_{p}}^{N_{p}} I_{n} S(z-\Delta z)
$$

where

$$
\begin{array}{rlrl}
S(z) & =A \frac{\sin [\beta(\Delta z-|z|)]}{\sin (\beta \Delta z)}, & |z| \leq \Delta z \\
& =0, \quad \text { otherwise, }
\end{array}
$$

and $A$ is a suitable normalization constant the choice of which will be discussed later. Equation (7) requires the discrete spectrum of this new representation. Note that a pulse of width equal to the sampling interval $\Delta z$ and amplitude $(1 / \Delta z)$ represents a delta function in the discrete sense. Therefore (8) can also be written as

$$
J_{\mathrm{PWC}}=\sum_{n=-N_{p}}^{N_{\rho}} I_{n} \delta(z-n \Delta z) \Delta z
$$


where $\delta$ denotes the delta function. It can be seen from (9) and (10) and Fig. 1 that $J_{\text {PWS }}$ can be written as

$$
J_{\mathrm{PWS}}=A \Delta z\left[S(z)^{*} \sum_{n=-N_{p}}^{N_{p}} I_{n} \delta(z-n \Delta z)\right]
$$

where the asterisk denotes the convolution operation. Therefore,

$$
\tilde{J}_{\mathrm{PWS}}=A \Delta z \tilde{S} \tilde{J}_{\mathrm{PWC}} \text {. }
$$

The spectrum of the current distribution represented in terms of PWS basis functions becomes a product of $\tilde{S}$ with the spectrum computed by the FFT. Note that no advantage would be gained if $\tilde{S}$ is also computed by the FFT. Since the DFT itself is an approximation to the Fourier transform, here we derive an improved approximation to the Fourier transform by computing $\tilde{S}$ in a closed form. The normalization constant is chosen so as to make the area of the pulse (shown shaded in Fig. 1) equal to unity, i.e., $A \Delta z=1$. The expression for $\tilde{S}$ can be written as

$$
\tilde{S}(f)=\frac{2 \beta[\cos (2 \pi f \Delta z)-\cos (\beta \Delta z)]}{\Delta z\left[\beta^{2}-(2 \pi f)^{2}\right] \sin (\beta \Delta z)} .
$$

Although it is of no interest as far as this paper is concerned, it may be mentioned that (12) follows readily from the principle of pattern multiplication. It is obvious that before computing the inverse transform, the spectrum must be multiplied by (1/ $\tilde{S})$ so that the inverse transform yields the amplitudes of the PWS basis functions. This can be easily incorporated in (7) by replacing $\tilde{D} \tilde{G}$ by $\tilde{D} \tilde{G} \tilde{S}$. Note that this multiplication need be done only once at the start of the iteration and no extra computational effort is required as the iteration proceeds. Other basis functions such as piecewise linear basis can also be incorporated in a similar manner.

\section{Two-Dimensional Current Distributions}

Having illustrated this method to a problem involving the current distribution in one dimension, we now consider its application to a two-dimensional current distribution, namely, scattering by a thin rectangular plate. A normally incident $(x$ directed) plane wave $E_{x i}(x, y)$ is considered. The relevant integrodifferential equations for a rectangular plate of dimensions $(2 a \times 2 b)$ located symmetrically in the $z=0$ plane of a Cartesian coordinate system are

$$
\begin{gathered}
{\left[\frac{\partial^{2}}{\partial x^{2}}+\beta^{2}\right] A_{x}(x, y)+\frac{\partial^{2}}{\partial x \partial y} A_{y}(x, y)} \\
=-j \omega \epsilon E_{x i}(x, y) \\
{\left[\frac{\partial^{2}}{\partial y^{2}}+\beta^{2}\right] A_{y}(x, y)+\frac{\partial^{2}}{\partial x \partial y} A_{x}(x, y)=0} \\
x \in[-a, a] \\
y \in[-b, b] \\
z=0
\end{gathered}
$$

where the components of the magnetic vector potential are given by

$$
\begin{aligned}
& A_{x}(x, y)=J_{x}(x, y) * G(x, y) \\
& A_{y}(x, y)=J_{y}(x, y)^{*} G(x, y) .
\end{aligned}
$$

$J_{x}$ and $J_{y}$ denote the current distributions along the $x$ - and $y$ directions and the Green's function is given by

$$
G\left(x-x^{\prime}, y-y^{\prime}\right)=\frac{\exp \left[j \beta \sqrt{\left(x-x^{\prime}\right)^{2}+\left(y-y^{\prime}\right)^{2}}\right]}{4 \pi \sqrt{\left(x-x^{\prime}\right)^{2}+\left(y-y^{\prime}\right)^{2}}} .
$$

Following the procedure discussed by Ko and Mittra [2], iterative expressions for the current distributions $J_{x}$ and $J_{y}$ can be readily obtained. However, due to reasons mentioned in Section II, the current distributions obtained would be in terms of piecewise constant basis functions.

For basis functions in this case we choose the ones employed by Newman and Pozar [11], i.e.,

$$
\begin{aligned}
S_{x}(x, y) & =\frac{A[\sin \beta(\Delta x-|x|)] \cos (\beta y)}{\sin (\beta \Delta x)}, \quad|x| \leq \Delta x \\
& |y| \leq \Delta y / 2 \\
& =0, \quad \text { otherwise }
\end{aligned}
$$

where $\Delta x$ and $\Delta y$ represent the sampling intervals in the $x$ and $y$ directions, respectively. The $x$-component of the current distribution is expressed in terms of these basis functions with overlap along the $x$-direction and no overlap along the $y$ direction. A similar form $\left(S_{y}(x, y)\right)$ is employed for the $y$ component of the current distribution. This representation of the current distribution is considered as a convolution of the current distribution represented in terms of PWC basis functions with $S_{x}(x, y)$ (for the $x$-component) and $S_{y}(x, y)$ (for the $y$-component). An expression for $\tilde{S_{x}}(f, g)$ can be written as

$$
\begin{aligned}
\tilde{S}_{x}(f, g)= & A \beta \Delta y[\cos (2 \pi f \Delta x)-\cos (\beta \Delta x)] \\
& \cdot[\operatorname{sinc}\{(\Delta y / 2)(\beta-2 \pi g)\} \\
& +\operatorname{sinc}\{(\Delta y / 2)(\beta+2 \pi g)\}] /\left[\left\{\beta^{2}\right.\right. \\
& \left.\left.-(2 \pi f)^{2}\right\} \sin (\beta \Delta x)\right]
\end{aligned}
$$

where the normalization constant $A=1 /(\Delta x \Delta y)$ and $f$ and $g$ denote the Fourier transform variables. A similar expression for $\tilde{S_{y}}(f, g)$ can be obtained by interchanging $x$ and $y$ and $f$ and $g$ in the above expression. The modified iterative expressions for $\tilde{J}_{x}$ and $\tilde{J}_{y}$ can be written as ([2] and (12)),

$$
\begin{aligned}
& \tilde{J}_{x}^{(k+1)}=\frac{2\left[\beta^{2}-(2 \pi f)^{2}\right]\left[-\widetilde{\theta E}_{x i}+\widetilde{\theta F}_{x}^{k}\right]}{\beta Z_{0} \tilde{S}_{x} \sqrt{\beta^{2}-(2 \pi f)^{2}-(2 \pi g)^{2}}} \\
& \tilde{J}_{y}^{(k+1)}=\frac{2[(2 \pi f)(2 \pi g)]\left[-\widetilde{\theta E_{x i}}+\widetilde{\tilde{\theta} F_{x}^{k}}\right]}{\beta Z_{0} \tilde{S}_{y} \sqrt{\beta^{2}-(2 \pi f)^{2}-(2 \pi g)^{2}}}
\end{aligned}
$$

where

$$
F_{x}^{k}=F^{-1}\left[( - j Z _ { 0 } / \beta ) \tilde { G } \left\{\left(\beta^{2}-(2 \pi f)^{2}\right) \tilde{J}_{x}^{k} \tilde{S}_{x}\right.\right.
$$




$$
\tilde{G}=\frac{j}{2 \sqrt{\beta^{2}+(2 \pi f)^{2}-(2 \pi g)^{2}}} .
$$

In $(20),(21)$, and (22), the operators $\theta$ and $\hat{\theta}$ denote twodimensional truncational operators, and the tilde denotes the two-dimensional Fourier transform. $\tilde{G}$ denotes the Fourier transform of the Green's function and $Z_{0}$ is the intrinsic impedance of free space.

\section{Numerical. Results and Discussion}

The effectiveness of the above approach is demonstrated in this section by comparing the results obtained using PWS basis functions with those obtained by the original SIT. It must be mentioned here that the word convergence is somewhat ambiguous. It can refer to the convergence of the iterative process itself or to the convergence of the results obtained by the iterative process. Since the convergence of general iterative methods has been discussed by Youla [12] and that of the SIT has also been discussed elsewhere [8], in what follows, convergence refers to the results obtained by the SIT unless explicitly stated.

We first consider the input impedance of a cylindrical antenna since this is a rigorous test parameter for the convergence of the solution. Since the method of solution is iterative, it is obvious that a good choice of the initial current distribution will significantly hasten the convergence of the iteration. The sinusoidal distribution is known to be an excellent approximation to the current distribution on thin wires. For this reason, the initial current distribution in all - examples illustrated was assumed to be sinusoidal in magnitude and of zero phase. Furthermore, an acceleration parameter [8] was also employed to hasten the convergence. A delta gap feed model has been assumed.

The input impedance versus the number of basis functions in representing the current distribution is plotted in Figs. 2-4 for wire lengths of $0.3 \lambda$, and $0.5 \lambda$, and $0.7 \lambda$. Convergence of moment method solutions as a function of both the number and types of basis functions have been well discussed [13], [14]. It is even observed [14] that for some wire lengths the input impedances appear to converge to different values with increasingly large number of basis functions for different types. Moreover when the impedances are plotted against $1 /$ $\left(2 N_{p}+1\right)$, the impedances appear to diverge as has been observed in [14]. The reasons for this behavior have been explained [14]. Therefore our results mainly illustrate the faster convergence of the SIT when a better approximation is employed in representing the current distribution. It may be seen from Fig. 2 that for the $0.3 \lambda$ case, due to the short length of the antenna, relatively few PWC basis functions may be good approximations to the current distribution and the gain in convergence with PWS basis functions may not be significant. In fact no difference is observed in the real part of the input impedance. However as the length increases, the advantages of the PWS representation become apparent (Figs. 3 and 4).

It has been mentioned in Section II that by the introduction of PWS basis functions we reduced the aliasing errors in the computation of the Fourier transform of the current distribution and the inverse Fourier transform of its spectrum. This is

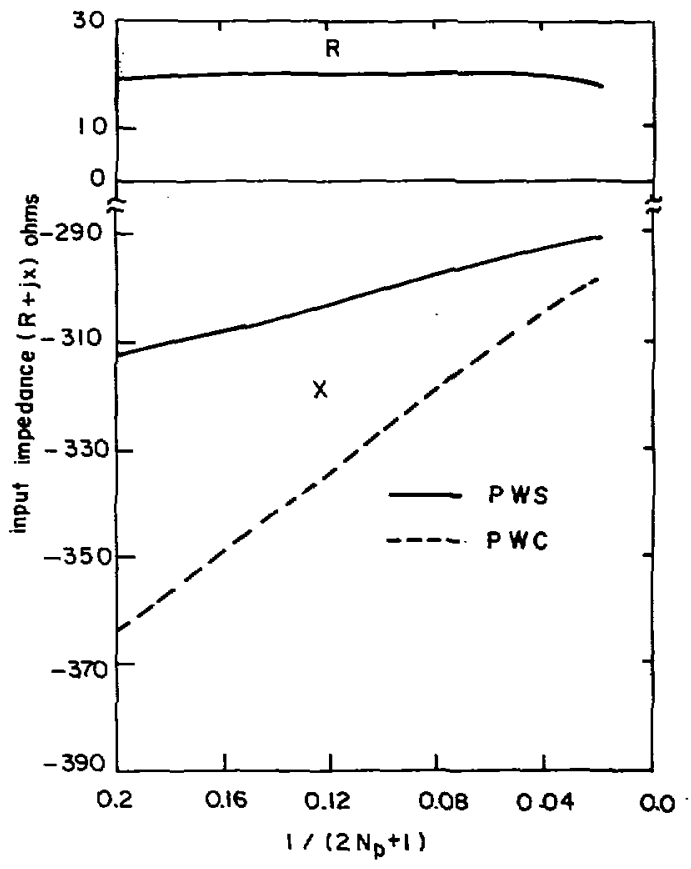

Fig. 2. Convergence of the input impedance. $2 h=0.3 \lambda, h / 2 a=100$.

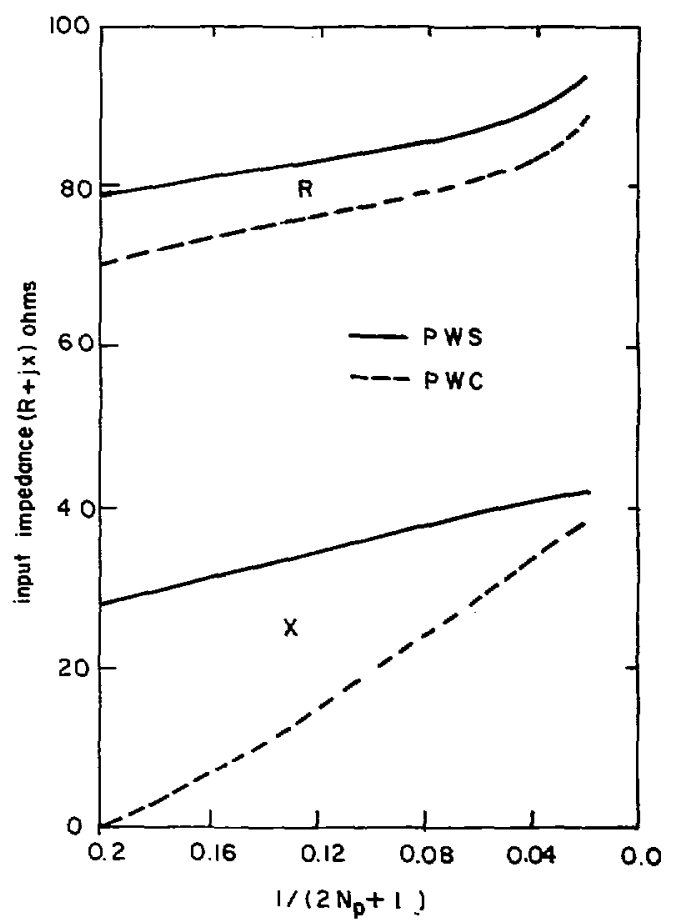

Fig. 3. Convergence of the input impedance. $2 h=0.5 \lambda, h / a=100$.

in view of the fact that the PWS representations is closer to the actual current distribution as compared to the PWC representation and consequently the error involved in the representation of the function itself is less, leading to a more accurate spectrum. This fact has been verified through several trial runs using different basis functions and computing the errors in the spectrum. However, the aliasing errors in the spectrum of the electric field and the Fourier transform of the truncated electric field (cases (c) and (d) in Section II) are still present. It may be mentioned here that a technique has been developed by 


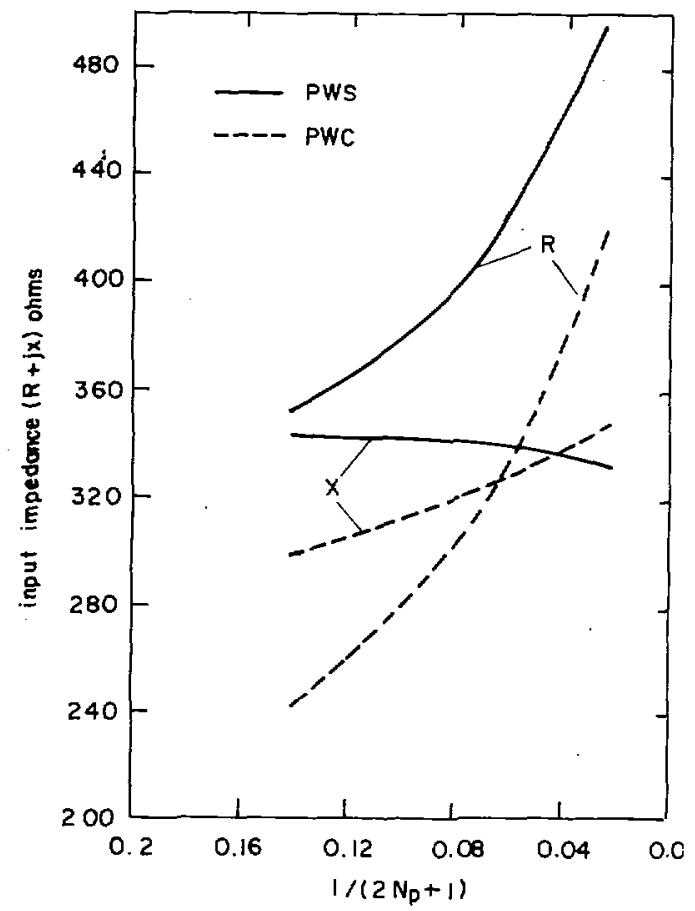

Fig. 4. Convergence of the input impedance, $2 h=0.7 \lambda, h / a=100$.

Narasimhan and Karthikeyan [15] to reduce the aliasing error in the computation of Fourier integrals by the FFT algorithm. They employ the trapezoidal rule for a more accurate computation of the Fourier integral. Their method can be derived from the one presented in this paper with a choice of piecewise linear functions as the basis functions. However, care must be taken in handling the function at its discontinuities. When a discrete delta function representation is convolved with a piecewise linear function, extra half-triangles would appear at the points of discontinuity. The spectrum of these half triangles must be subtracted from the total spectrum so as to result in a more accurate spectrum. Their method, besides requiring two FFT computation does not permit straight forward evaluation of the inverse Fourier transform. That is, given an accurate spectrum, computation of the function represented in terms of piecewise linear basis functions becomes difficult especially when the function is discontinuous such as the truncated electric field arising in the SIT. Moreover, in a method like SIT where repeated evaluation of the Fourier transform is required (three times per iteration), the additional computational expense may not be entirely justifiable. For this reason, the aliasing errors in cases (c) and (d) (Section II) are difficult to minimize without additional computational expense. In contrast, with the approach followed in this paper, the expression $\tilde{D} \tilde{G} \tilde{S}((7)$ and (12)) need be computed only once, and no extra computations as compared to the original SIT are incurred as the iteration proceeds.

In order to compare this method with other methods we choose point matching at one end (since this technique requires a relatively large number of subdomain basis functions for a given degree of accuracy) and reaction matching at the other end as an example of full surface testing (since reaction matching with PWS basis functions has the unique feature that

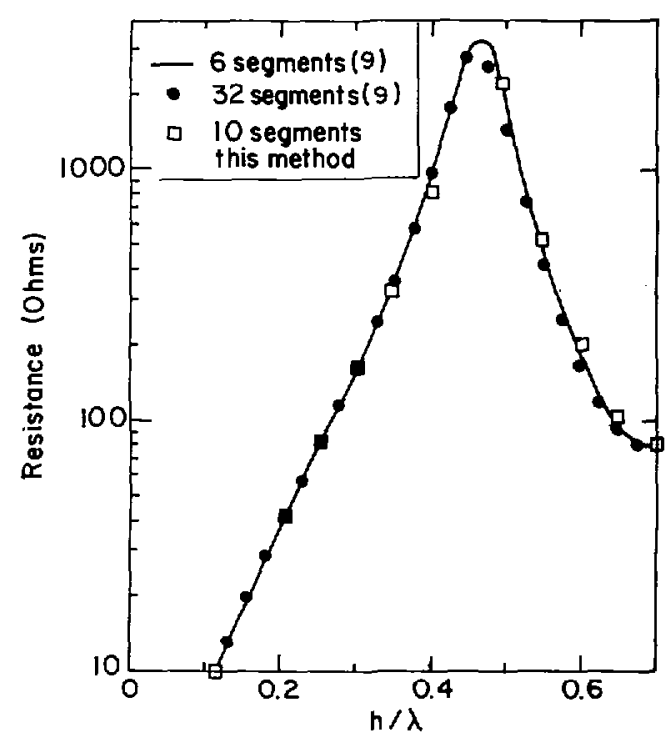

Fig. 5. Input resistance as a function of the wire length, $h / a=2000$.

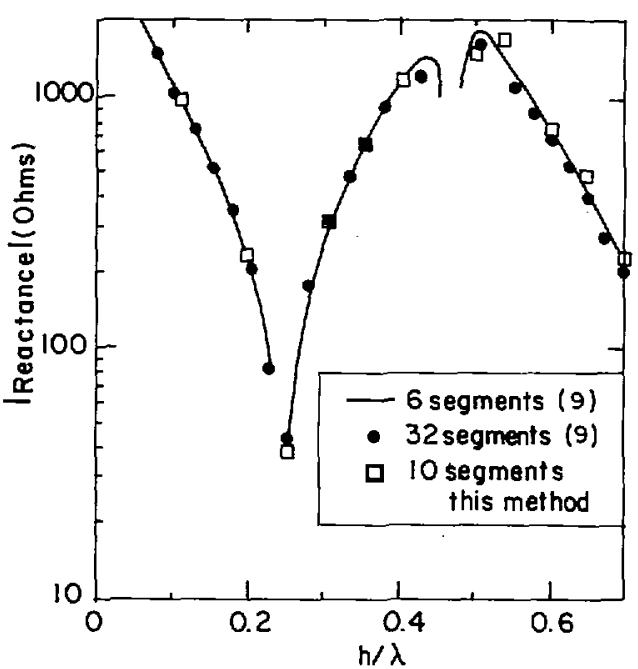

Fig. 6. Input reactance as a function of the wire length, $h / a=2000$.

for some problems all the integrations required can be evaluated in a closed form). In Figs. 5 and 6, the input impedance as a function of the wire length with nine PWS basis functions is shown. This is also compared with the results obtained by Richmond [9] with five PWS basis functions and reaction matching and that obtained by Harrington [9] with 31 piecewise linear basis functions and point matching. Due to reasons mentioned in the above paragraph, the SIT even with PWS basis functions need not be expected to yield results as rapidly convergent as those obtained by reaction matching. However, with a slightly larger number of basis functions, the calculated values of input impedance are seen to compare well with other results. Thus spectral matching with PWS basis functions serves as a compromise between point matching and reaction matching as far as the number of basis functions are concerned. It, however, has computational advantages for large problems.

Application of this technique to a problem of scattering by a thin square plate is illustrated in Fig. 7. Here we choose the 


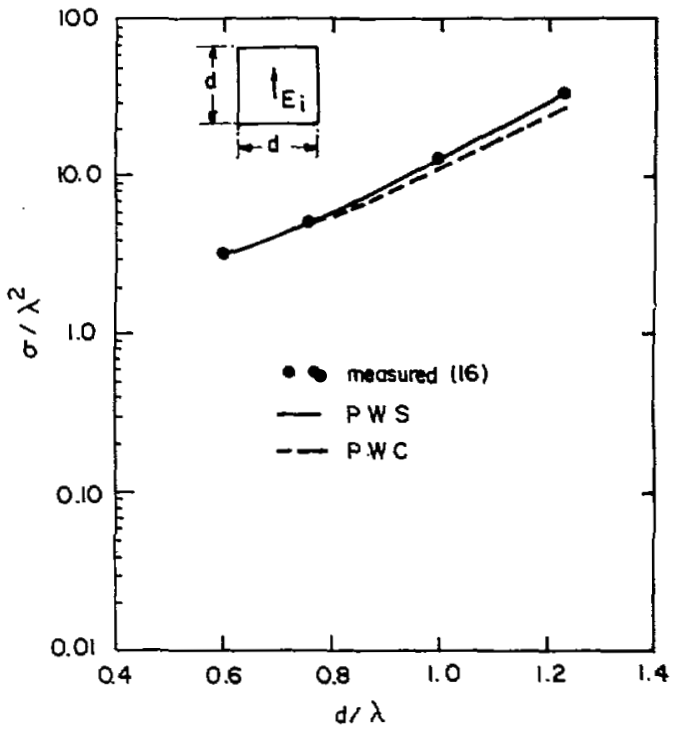

Fig. 7. Monostatic radar cross section as a function of plate size for normal incidence.

scattering cross section as the parameter for comparing the convergence of PWS and PWC basis functions. Comparison is also made with the experimental results of Kouyoumjian [16]. Note that the scattering cross section being far-field dependent is not as stringent a test parameter as the input impedance. A minimum of 35 basis functions were employed for the results illustrated in Fig. 7. As is to be expected, the improvement in scattering cross section is significant for plates of larger dimensions, since relatively few PWC basis functions may be sufficient to represent the current distribution on small plates. We have also investigated PWS basis functions without the cos $(\beta y)$ term in (19). No significant difference was observed. The current distribution and the total tangential electric field on a 1 $\lambda \times 1 \lambda$ square plate is shown in Figs. 8 and 9. The current distribution has been compared with the results of Glisson [17]. As is to be expected with lesser number of basis functions, the current distribution is in a close agreement with the more accurate results of Glisson except for the singular portion near the edges.

In both the above illustrations, the initial current distribution was assumed to be sinusoidal. Although the computational advantages of the SIT can be fully realized when it is used as a hybrid method [2], yet we find that in most cases convergence was achieved within ten iterations with the use of the acceleration parameter. It can also be seen from (13) and (20) that as the pulse widths $\Delta z$ (in the one-dimensional case) and $\Delta x$ and $\Delta y$ (in the two-dimensional case) are reduced, the spectrum $\tilde{S}$ becomes nearly unity and the results obtained using PWS basis functions will not be largely different from those obtained using PWC basis functions. It is interesting to observe that if the sampling rate is sufficiently high, the results obtained would be independent of the type of basis functions that are used to represent the current distribution. Thus, the advantages of the technique presented in this paper will be significant as long as $\Delta z$ or $\Delta x$ and $\Delta y$ are not too small.

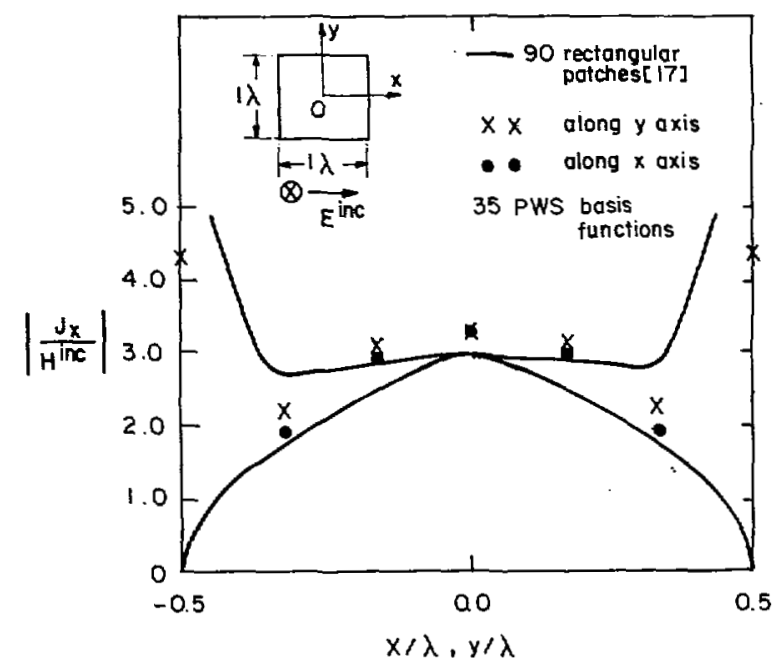

Fig. 8. Current distribution on a $1 \lambda \times 1 \lambda$ square plate for a normally incident plane wave.

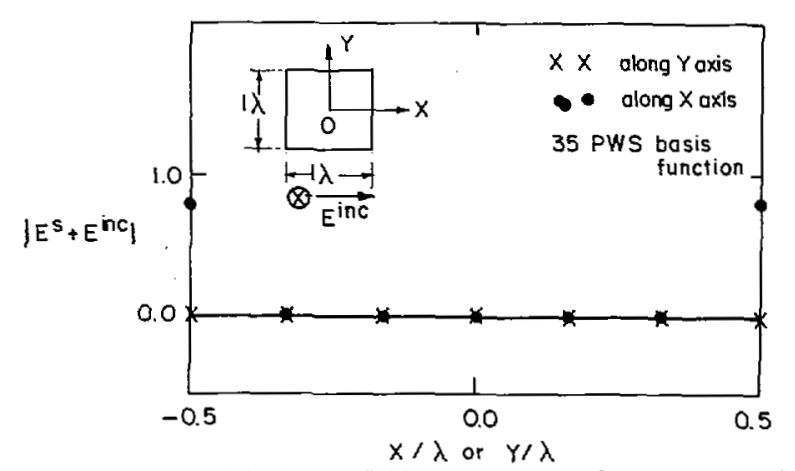

Fig. 9. Total tangential electric field on a $1 \lambda \times 1 \lambda$ square plate for a normally incident plane wave.

\section{CONCLUSION}

It has been shown that the convergence of the results obtained by the spectral iteration technique could be improved significantly by incorporating more appropriate basis functions to represent the current distribution. A technique which implements this modification with a minimum of extra computational effort while retaining all the advantages of the SIT has been presented. Results of input impedance for wire antennas and scattering cross section of flat plates with piecewise sinusoidal functions as the basis functions in the representation of the current distribution illustrate the validity of the method. Furthermore, it has been observed that with this method the convergence of the original SIT does not deteriorate. In fact, in some examples it has even rendered a nonconvergent iteration convergent and in some cases improved the rate of convergence. It has also been discussed in [8] that the SIT remains largely convergent when the sampling interval is not too small. Since the method presented in this paper works best when the sampling interval is not too small, the gain in the convergence of the results obtained will be significant. This modification is seen to further extend the capabilities of the SIT in handling moderate to large size 
problems. It has been shown that as far as the convergence of the results obtained by this technique as a function of the number or basis functions is concerned, it is a compromise between reaction matching and point matching. With the help of the stacked SIT algorithm [6] this technique can also be extended to more complex wire and surface geometries.

\section{REFERENCES}

[1] G. A. Thiele, "An overview of methods which combine the Moment method with eigenfunctions and asymptotic techniques," Ohio State Univ. Electrosci. Lab. Rep., 1978.

[2] W. L. Ko and R. Mittra, "A new approach based on a combination of integral equation and asymptotic techniques for solving electromagnetic scattering problems," IEEE Trans. Antennas Propagat., vol. AP-25, pp. 187-197, Mar. 1977.

[3] T. K. Sarkar and S. M. Rao, "The application of the conjugate gradient method for the solution of electromagnetic scattering from arbitrarily oriented wire antennas," IEEE Trans. Antennas Propagat., vol. AP32, pp. 398-403, Apr. 1984.

[4] B. T. Borup and O. M. P. Gandhi, "Fast Fourier Transform method for calulation of SAR distributions in finely discretized inhomogeneous models of biological bodies," IEEE Trans. Microwave Theory Tech., vol. MTT-32, pp. 355-360, Apr. 1984.

[5] R. Mittra, W. L. Ko, and Y. Rahmat-Samii, "Transform approach to electromagnetic scattering," Proc. IEEE, vol. 67, pp. 1486-1503, Nov. 1979.

[6] R. Kastener and R. Mittra, "A spectral iteration technique for analyzing scattering from arbitrary bodies, Part I: Cylindrical scatterers with E-wave incidence," IEEE Trans. Antennas Propagat., vol. AP31, pp. 499-506, May 1983.

[7] J. W. Cooley, P. A. Lewis, and P. D. Welch, "Application of the FFT to the computation of Fourier integrals, Fourier series and convolution integrals," IEEE Trans. Audio Eletroacoust., vol. AU-15, pp. 7984, Jan 1967.

[8] S. A. Bokhari and N. Balakrishnan, "Analysis of cylindrical anten-
nas-A spectral iteration technique," IEEE Trans. Antennas Propagat., vol. AP-33, pp. 251-258, Mar. 1985.

[9] G. A.Thiele, "Wire antennas," in Computer Techniques for Electromagnetics, R. Mittra, Ed. Oxford: Pergamon, 1973.

[10] E. H. Newman and D. M. Pozar, "Considerations for efficient wire/ surface modeling," IEEE Trans. Antennas Propagat., vol. AP-28, pp. 121-125, Jan. 1980.

[11] E. H. Newman and D. M. Pozar, "Electromagnetic modelling of composite wire and surface geometries," IEEE Trans. Antennas Propagat., vol. AP-26, pp. 784-789, 1978.

[12] D. C. Youla, "Generalized image restoration by the method of alternating orthogonal projections," IEEE Trans. Circuits Syst., vol. CAS-25, pp. 694-702, Sept. 1978.

[13] E. K. Miller and F. J. Deadrick, "Some computational aspects of thin wire modelling," in Numerical and Asymptotic Techniques in Electromagnetics, R. Mittra, Ed. New York: Springer-Verlag, 1975.

[14] R. Mittra and C. A. Klein, "Stability and covergence of moment method solutions," in Numerical and Asymptotic Techniques in Electromagnetics, R. Mittra, Ed. New York: Springer-Verlag, 1975.

[15] M. S. Narasimhan and K. Karthikeyan, "Evaluation of Fourier transform integrals using FFT with improved accuracy and its applications," IEEE Trans. Antennas Propagat., vol. AP-32, pp. 404-408, Apr. 1984.

[16] R. G. Kouyoumjian, "The calculation of echo areas of perfectly conducting objects by the variational method," Ph.D. dissertation, Ohio State Univ., 1953.

[17] S. M. Rao, D. R. Wilton, and A. W. Glisson, "Electromagnetic scattering by surfaces of arbitrary shape," IEEE Trans. Antennas Propagat., vol. AP-30, pp. 409-418, May 1982.

S. A. Bokhari, for a photograph and biography please see page 258 of the March 1985 issue of this TRANSACTIONS.

N. Balakrishnan, for a photograph and biography please see page 258 of the March 1985 issue of this TRANSACTIONS. 